\title{
Instability of mRNA expression signatures of drug transporters in chronic myeloid leukemia patients resistant to imatinib
}

\author{
MARTA GROMICHO $^{1}$, MARTA MAGALHÃES ${ }^{1}$, FÁTIMA TORRES $^{2}$, JOANA DINIS ${ }^{1}$, \\ ALEXANDRA R. FERNANDES ${ }^{3-5}$, PAULA RENDEIRO ${ }^{2}$, PURIFICAÇÃO TAVARES ${ }^{2}$, \\ ANTÓNIO LAIRES ${ }^{1}$, JOSÉ RUEFF ${ }^{1}$ and ANTÓNIO SEBASTIÃO RODRIGUES ${ }^{1}$ \\ ${ }^{1}$ Human Molecular Genetics Research Centre (CIGMH), Department of Genetics, Faculty of Medical Sciences, \\ New University of Lisbon, Lisbon; ${ }^{2} \mathrm{CGC}$ (Clinical Genetics Center), Porto; ${ }^{3}$ Department of Life Sciences, \\ Faculty of Sciences and Technology, New University of Lisbon, Lisbon; ${ }^{4}$ Faculty of Biomedical Sciences, \\ Lusophone University of Humanities and Technology, Lisbon; ${ }^{5}$ Structural Chemistry Center, \\ Higher Technical Institute (IST), Lisbon, Portugal
}

Received June 29, 2012; Accepted September 7, 2012

DOI: 10.3892/or.2012.2153

\begin{abstract}
Despite the success of imatinib mesylate (IM) in the treatment of chronic myeloid leukemia (CML), approximately $30 \%$ of patients are resistant to therapy, mostly due to unknown causes. To profile the expression signatures of drug transporters throughout IM therapy and correlate them with resistance, we quantified mRNA expression levels of the $S L C 22 A 12, A B C B 1, A B C C 1, A B C G 2$ and $M V P$ genes in consecutive samples from peripheral blood or bone marrow of CML patients who were either responsive or resistant to IM. Additionally we identified and quantified $B C R$ - $A B L 1$ transcript variants and analyzed $1236 \mathrm{~T}>\mathrm{C} A B C B 1$ and $480 \mathrm{G}>\mathrm{C}$ $S L C 22 A 1$ polymorphisms. A relationship between the type of $B C R-A B L 1$ transcript or $A B C B 1$ or $S L C 22 A 1$ genotype and response to treatment was not discovered. However, the studied genes had higher expression levels in follow-up compared to the diagnostic samples, demonstrating a possible induction in expression. IM-sensitive patients presented significantly higher values of SLC22Al expression, suggesting higher drug influx. Most importantly, while responding patients demonstrated stable expression signatures in consecutive samples, there was
\end{abstract}

Correspondence to: Dr Marta Gromicho, Human Molecular Genetics Research Centre (CIGMH), Department of Genetics, Faculty of Medical Sciences, New University of Lisbon, Rua da Junqueira 100, 1349-008 Lisbon, Portugal

E-mail: marta.gromicho@fcm.unl.pt

Abbreviations: CML, chronic myeloid leukemia; IM, imatinib mesylate; MDR, multidrug resistance; MRPs, multidrug resistanceassociated proteins; qRT-PCR, quantitative real-time polymerase chain reaction; $\mathrm{Ct}$, threshold cycle; TKIs, tyrosine kinase inhibitors; $\mathrm{CCyR}$, complete cytogenetic response; MMR, major molecular response

Key words: chronic myeloid leukemia, $B C R-A B L 1$, drug resistance, imatinib, tyrosine kinase inhibitors, drug transporters, $A B C B 1$, $A B C C 1, A B C G 2, M V P, S L C 22 A 1$ considerable variation in IM-resistant patients, indicating that single point sampling expression signatures are not reliable in predicting clinical outcomes or prognostic features in these patients. Studies that assessed consecutive samples from CML patients in order to evaluate the variation in expression levels of transporter genes are limited yet our study emphasizes the importance of such approaches.

\section{Introduction}

Chronic myeloid leukemia (CML) is a myeloproliferative disease characterized by the malignant expansion of stem cells of myeloid origin in the bone marrow. It is associated with a characteristic chromosomal translocation, $\mathrm{t}(9 ; 22)$ (q34;q11), which results in an abnormal chromosome 22 called Philadelphia chromosome $(\mathrm{Ph})$ resulting in a fusion gene, BCR-ABL1 that codes for a protein with constitutive tyrosine kinase activity (reviewed in ref. 1).

Imatinib mesylate (IM) is the current standard treatment for $\mathrm{Ph}^{+} \mathrm{CML}$ (2). IM competitively binds to the ATP binding site in BCR-ABL1, maintaining the protein in its inactive form which leads to apoptosis of $\mathrm{Ph}^{+}$cells. In a 6-year follow-up study, $82 \%$ of CML patients obtained a complete cytogenetic response (CCyR) and 93\% did not exhibit progression while being treated with IM (3). However, recent evaluations with non-selected patients demonstrated that approximately $30 \%$ of patients exhibit suboptimal response or treatment failure (3-5), i.e. do not have an optimal response to IM therapy, defined as CCyR at 12 months (no $\mathrm{Ph}^{+}$cells) and major molecular response (MMR; BCR-ABL1:ABL1 $\leq 0.1 \%$ ) at 18 months (6). The second-line options for patients that require alternative therapies to standard-dose IM (400 mg daily) are: IM dose escalation (600-800 mg daily) or the second generation tyrosine kinase inhibitors (TKIs), dasatinib and nilotinib (2). However, resistance to these TKIs also occurs, and patients proceed to advanced disease or blast crisis, for which existing therapies are limited. Thus, resistance to TKIs is an increasingly important clinical problem. 
Mutations in the kinase domain of $B C R-A B L 1$ are responsible for approximately $40 \%$ of all cases of resistance, but in a number of patients the causes of resistance remain unclear (7). Adequate IM plasma level is an important feature for a satisfactory clinical response $(8,9)$ and factors that may affect IM intracellular action, such as drug absorption and extrusion, may impact the ability to achieve a maximal therapeutic benefit. Influx of IM is mediated by the human organic cation transporter 1 transporter ( $\mathrm{OCCTl}, S L C 22 \mathrm{Al})$ which is likely to play an important role in IM resistance (10-12) since CML patients with a higher $S L C 22 \mathrm{Al}$ expression before treatment had better results in terms of CCyR rates when treated with IM (13).

Furthermore, resistance may also be associated with the altered expression of ATP-binding cassette (ABC) family of transporters on cell membrane, the most common cause of multidrug resistance (MDR) (14). Among them, ABCB1 (MDR1, P-gp), ABCC1 (MRP1) and ABCG2 (BCRP) have been extensively related to MDR in leukemia $(15,16)$. Additionally, other proteins have also been associated with the MDR phenotype, such as the major vault protein (MVP) or the lung resistance protein (LRP) (17).

Few studies have been conducted in samples from CML patients focusing on MDR proteins and their implications in treatment response $(13,18,19)$. Recent results from our group demonstrated that during the development of resistance to increasing doses of IM, K562 CML cells overexpress several transporters (ABCB1,ABCC1,ABCG2,SLC22A1 and $M V P$ ), but we also observed that the expression pattern of the transporters was dynamic, varying with drug exposure (20). These results raised the hypothesis that patients treated with IM may also present variations in the expression of transporters during therapy, which may impact their response. To our knowledge, studies assessing consecutive samples from CML patients in order to evaluate the variation in expression levels of transporter genes do not exist. Thus, we quantified the gene expression levels of $S L C 22 A 1, A B C B 1, A B C C 1, A B C G 2$ and $M V P$ at several time points during treatment in $28 \mathrm{CML}$ patients who were either responsive or resistant to IM.

\section{Materials and methods}

Patients. Thirty-three adult patients, 17 male and 16 female with a median age of 57 years (range 20-77) diagnosed between 1984 and 2008 with $\mathrm{CML} \mathrm{Ph}^{+}$were enrolled in this study, which was performed in accordance with the Declaration of Helsinki. The samples were collected and sent to Clinical Genetics Center (CGC), Porto, Portugal, for diagnostic purposes. The genetic testing requisition for the molecular diagnostic tests included an informed consent as well as an authorization for the biological samples to be used for scientific research purposes. These documents were signed by the requesting physician and the patients. All samples included in this study were anonymized in order to protect patient confidentiality and to avoid traceability to other medical records.

The diagnosis of CML was based on standard clinical data and confirmed by cytogenetic and molecular analysis and response criteria were the same as the criteria defined by the European LeukemiaNet (6). Distinct groups were categorized according to their response to IM treatment: 15 were susceptible patients ( $\mathrm{S}, \mathrm{n}=15), 8$ were resistant without identified mutations in the kinase domain of BCR-ABL1 $(\mathrm{R}, \mathrm{n}=8)$ and 10 were resistant with mutations in the kinase domain of BCR-ABL1 $(\mathrm{RM}, \mathrm{n}=10)$. Most patients began IM therapy shortly after diagnosis. However, 5 patients (S-2, R-4, RM-1, RM-2 and RM-4) received prior therapy with hydroxyurea and/or interferon- $\alpha$.

For the 28 patients studied, the follow-up time ranged from 2 to 73 months, with a median observation of 46 months, and peripheral blood $(n=60)$ or bone marrow samples $(n=22)$ were collected with a median periodicity of six months. The cut-off period was March 2010. The date of diagnosis was considered time zero $(t=0)$, and the follow-up samples were identified according to the months after diagnosis $(t)$ in which the sample was collected (example, $t=12$, sample collected twelve months after diagnosis). The mononuclear cells were separated by gradient centrifugation, and guanidinium thiocyanate cell lysates were maintained at $-20^{\circ} \mathrm{C}$ until further use.

RNA isolation and cDNA synthesis. RNA was extracted using an RNeasy kit (Qiagen, Hilden, Germany) and stored at $-80^{\circ} \mathrm{C}$ until use. The concentration and purity of resulting RNA were estimated at 260 and $280 \mathrm{~nm}$ using the NanoDrop spectrophotometer ND-1000 (Thermo Scientific, Waltham, MA, USA), and only those samples with A260 to A280 ratios between 1.9 and 2.1 were further considered. Total RNA $(0.5 \mu \mathrm{g})$ was reverse transcribed with the High Capacity RNA-to-cDNA kit (Applied Biosystems, Foster City, CA, USA) in a final volume of $20 \mu 1$ according to the manufacturer's instructions.

BCR-ABL1 fusion transcript analysis and quantification. Fusion gene transcript analysis was performed as previously described (21-23). BCR-ABL1 transcripts were quantified using the BCR-ABL1 Mbcr FusionQuant kit for the real-time quantitative PCR analysis of BCR-ABL Mbcr p210 transcripts (ref. no. FQPP-10-CE, Ipsogen, Marseille, France) according to the manufacturer's instructions and standardized protocol (23).

Mutation analysis. A fragment corresponding to amino acids 225 to 498 of the $B C R-A B L 1$ kinase domain was amplified from cDNA in a nested PCR. After purification of PCR products through QIAquick columns (Qiagen), standard dideoxy chain-termination DNA sequencing was performed in the forward and reverse directions using an ABI PRISM BigDye Terminator on an automated ABI PRISM 3100 Avant Genetic Analyzer (Applied Biosystems). The direct sequences were analyzed using sequence analysis software V3.3 and the SeqScape software V2 (Applied Biosystems) and compared with the GeneBank NM_007313.2.ABL1 sequence.

Real-time quantitative PCR ( $q R T$ - $P C R)$. The measurement of mRNA levels of the $A B C B 1, A B C C 1, A B C G 2$, SLC22A1 and $M V P$ genes was based on SYBR1 Green PCR master mix (Applied Biosystems) and melting curve analysis using the 7300 Real-Time PCR system (Applied Biosystems). The following Assay-on-Demand products from Applied Biosystems used were: $A B C B 1$, Hs00184491_m1; $A B C C 1$, Hs00219905_m1; ABCG2, Hs00184979_m1; LRP/MVP, Hs00245438_m1; SLC22A1, Hs00427550_m1 and GAPDH, $4352934 \mathrm{E}$ was used as the control gene. 


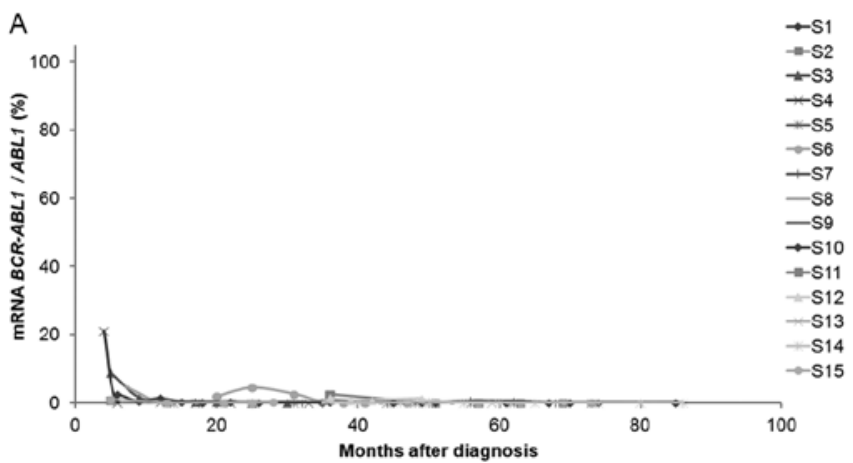

B
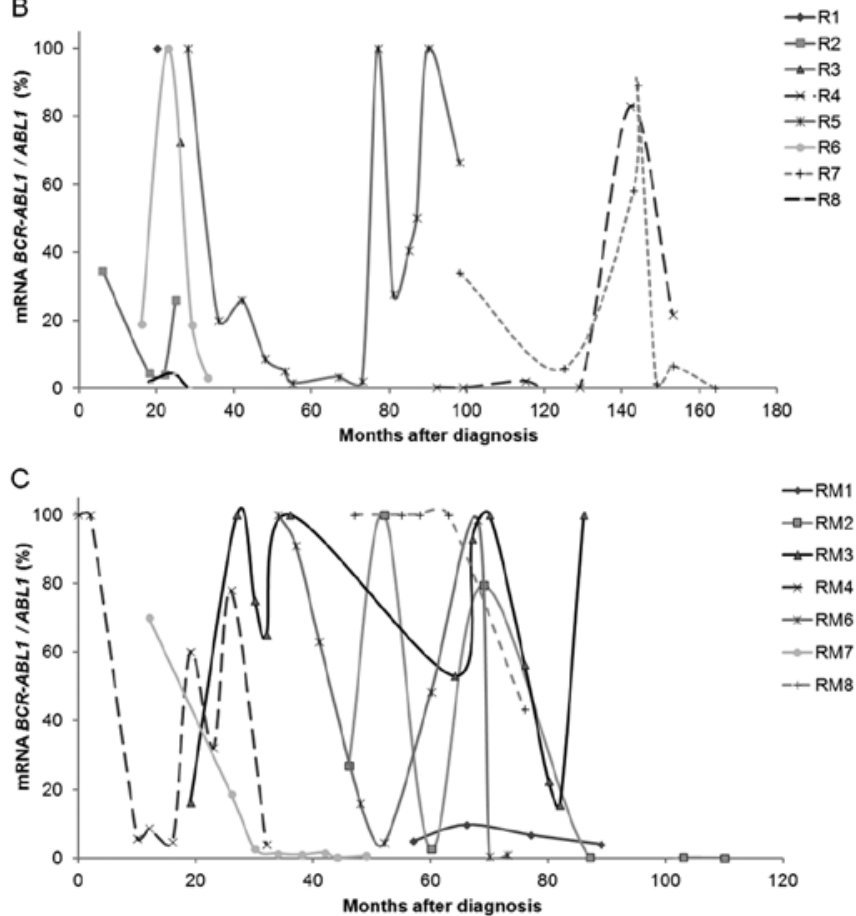

Figure 1. Values of $B C R-A B L 1$ expression (calculated as the percentage of $B C R-A B L 1 / A B L 1$ ) over a period of time. (A) Patients sensitive to IM (S), (B) patients resistant to IM (R), (C) patients resistant to IM with identified mutations in the kinase domain of $B C R-A B L 1(R M)$.

Template controls and reverse transcriptase controls (RT negative) for each cDNA synthesis were included. Only those that did not amplify, showing that primer-dimer formation and genomic DNA contamination were negligible, were further considered.

The mean values of the triplicate qRT-PCR reactions for each assay were normalized with the expression values for each gene obtained for the calibrator sample, the K562 cell line. Then, the relative gene expression levels were calculated using the equation $\left[\left(1+\mathrm{E}_{\text {targe }}\right)^{-\Delta \mathrm{Ct} \text { (target) }}\right] /\left[\left(1+\mathrm{E}_{\text {reference }}\right)^{-\Delta \mathrm{Ct}(\text { reference })}\right]$ where $\mathrm{E}$ is the value of efficiency of the qRT-PCR reaction for each assay, target refers to the values obtained for the analyzed gene and reference relates to the values obtained for the gene used to normalize the relative expression values (24). Values were reported as an average of triplicate/duplicate analysis.

K562 cell line. The human CML cell line, K562, expressing $B C R-A B L 1$ was used as a calibrator for the quantitative analysis of gene expression. The K562 cell line was maintained in RPMI-1640 medium (Sigma-Aldrich, St. Louis, MO,
USA) with $10 \%$ fetal bovine serum (Sigma-Aldrich) and $1 \%$ penicillin-streptomycin (Sigma-Aldrich) at $37^{\circ} \mathrm{C}$ under a $5 \%$ $\mathrm{CO}_{2}$ atmosphere.

Statistical/data analysis. The non-parametric Mann-Whitney test was applied to analyze differences in the levels of expression of each gene between the group of patients susceptible to IM and the group of patients resistant to the therapy. The correlation between the expression values of the genes studied was determined by the Spearman's correlation coefficient. The level of significance was set at $\mathrm{p}<0.05$ in all analyses. All statistical tests were performed using SPSS software, version 19.0.

\section{Results}

Patient samples. We analyzed the expression levels of the genes $A B C B 1, A B C C 1, A B C G 2, S L C 22 A 1$ and $M V P$ over a period of time in 82 samples corresponding to a total of 28 patients, 14 sensitive and 14 resistant to IM ( 7 with and 7 without mutations in $B C R-A B L 1$ ) in order to elucidate the role of the genes in the response to treatment. Both transcript variant analysis and polymorphism analysis were performed for 33 patients.

In both groups of non-responder patients ( $\mathrm{R}$ and $\mathrm{RM}$ ) comprising those that did not achieve an optimal cytogenetic or molecular response following treatment under $400 \mathrm{mg} /$ day of IM, according to European LeukemiaNet recommendations, a number of patients received higher doses of IM (R5) and/or changed to dasatinib (R-7, RM-1, RM-2, RM-3, RM-4, RM-7). Several of these patients subsequently achieved CCyR and/or MMR (RM-1, RM-2, RM-4) whereas the others maintained a suboptimal response or failure even after therapy change.

Transcript variants. Sixteen patients carried the e14a2 BCR-ABL1 rearrangement (48.5\%); eleven, the e13a2-BCRABL1 (33.3\%); one patient, both e14a2 and e13a2 (3.0\%); three patients, the e13a2 and ela $2(9.1 \%)$; and two patients, e14a2 and ela2 (6.1\%) (data not shown). No relationship between the type of BCR-ABL1 transcript and response to treatment was detected (Fisher's test, $\mathrm{p}>0.05$ ).

Transcript expression of $B C R-A B L 1$ over time. At the time of diagnosis both patients susceptible and resistant to IM presented high values of $B C R-A B L 1$ expression. Over time that expression changed to null or very low values in patients responding to IM (MMR, BCR-ABL1:ABL1 $\leq 0.1 \%$ at 18 months), as expected. However, for patients resistant to therapy, the expression of $B C R-A B L 1$ varied greatly over time; even when the values were reduced to undetectable levels, the expression increased again over time translating into acquired resistance (Fig. 1). As expected, in follow-up samples, patients susceptible to therapy presented significantly lower values of $B C R-A B L 1$ expression (S vs. R, p<0.001; S vs. RM, p<0.001).

Transcript expression of $A B C B 1, A B C C 1, A B C G 2, S L C 22 A 1$ and MVP in diagnostic and follow-up samples. The expression levels of $A B C B 1, A B C C 1, A B C G 2, S L C 22 A 1$ and $M V P$ were higher in follow-up samples compared to the diagnostic samples (Table I). The increases in the expression level from the diagnostic to the follow-up sample were significant in samples from sensitive patients for $A B C B 1$ ( $\mathrm{p}=0.001), A B C C 1$ 
Table I. Relative expression values of the studied genes.

\begin{tabular}{|c|c|c|c|c|c|c|}
\hline \multirow{2}{*}{\multicolumn{2}{|c|}{ Sample }} & \multicolumn{5}{|c|}{ Gene } \\
\hline & & $A B C B 1$ & $A B C C 1$ & $A B C G 2$ & $M V P$ & $S L C 22 A 1$ \\
\hline \multicolumn{7}{|c|}{ Sensitive (S) } \\
\hline \multirow{5}{*}{1} & $\mathrm{~A}(\mathrm{~PB})$ & 0.70 & 0.27 & 0.21 & 15.05 & 2.82 \\
\hline & $\mathrm{C}(\mathrm{PB})$ & 7.37 & 0.64 & 0.33 & 64.16 & 18.69 \\
\hline & $\mathrm{F}(\mathrm{PB})$ & 6.23 & 0.89 & 0.21 & 60.45 & 21.00 \\
\hline & $\mathrm{I}(\mathrm{PB})$ & 4.56 & 0.72 & 0.14 & 37.45 & 6.39 \\
\hline & $\mathrm{K}(\mathrm{PB})$ & 4.77 & 0.93 & 0.43 & 42.40 & 11.76 \\
\hline \multirow[t]{2}{*}{2} & Dx (BM) & 0.00 & 0.08 & 0.00 & 6.77 & 0.00 \\
\hline & $\mathrm{A}(\mathrm{BM})$ & 0.70 & 0.17 & 0.20 & 13.61 & 0.51 \\
\hline \multirow[t]{4}{*}{3} & $\mathrm{~A}(\mathrm{~PB})$ & 13.18 & 1.08 & 0.17 & 59.52 & 10.67 \\
\hline & $\mathrm{B}(\mathrm{PB})$ & 9.56 & 0.92 & 0.25 & 40.03 & 8.89 \\
\hline & $\mathrm{D}(\mathrm{BM})$ & 14.19 & 0.77 & 0.19 & 52.37 & 12.82 \\
\hline & $\mathrm{G}(\mathrm{BM})$ & 13.24 & 0.87 & 0.17 & 66.65 & 20.46 \\
\hline \multirow[t]{2}{*}{4} & $\mathrm{~A}(\mathrm{BM})$ & 0.63 & 0.12 & 0.04 & 8.30 & 0.14 \\
\hline & $\mathrm{F}(\mathrm{PB})$ & 11.69 & 1.74 & 0.62 & 46.56 & 5.26 \\
\hline \multirow[t]{4}{*}{5} & $\mathrm{~B}(\mathrm{~PB})$ & 7.38 & 0.65 & 0.24 & 84.02 & 9.28 \\
\hline & $\mathrm{F}(\mathrm{PB})$ & 27.93 & 1.22 & 0.61 & 49.96 & 19.40 \\
\hline & $\mathrm{H}(\mathrm{PB})$ & 6.20 & 0.84 & 0.28 & 97.01 & 13.28 \\
\hline & $\mathrm{J}(\mathrm{PB})$ & 12.38 & 1.11 & 0.48 & 107.98 & 13.66 \\
\hline 6 & $\mathrm{I}(\mathrm{PB})$ & 13.48 & 0.82 & 0.53 & 97.58 & 15.05 \\
\hline \multirow[t]{2}{*}{7} & $\mathrm{D}(\mathrm{PB})$ & 6.44 & 1.34 & 0.00 & 218.54 & 51.07 \\
\hline & I (PB) & 0.00 & 0.17 & 0.28 & 61.73 & 10.96 \\
\hline \multirow[t]{6}{*}{8} & $\mathrm{Dx}(\mathrm{PB})$ & 0.00 & 0.33 & 0.00 & 51.36 & 8.30 \\
\hline & $\mathrm{B}(\mathrm{PB})$ & 11.88 & 0.81 & 0.56 & 102.26 & 15.71 \\
\hline & $\mathrm{E}(\mathrm{PB})$ & 9.78 & 0.74 & 0.78 & 73.38 & 9.72 \\
\hline & $\mathrm{G}(\mathrm{BM})$ & 7.49 & 0.62 & 1.46 & 47.16 & 7.52 \\
\hline & $\mathrm{I}(\mathrm{PB})$ & 5.92 & 1.08 & 0.82 & 51.89 & 14.70 \\
\hline & $\mathrm{M}(\mathrm{PB})$ & 5.28 & 0.73 & 3.24 & 41.29 & 8.94 \\
\hline 9 & $\mathrm{Dx}(\mathrm{BM})$ & 0.00 & 0.17 & 0.00 & 9.24 & 1.10 \\
\hline \multirow[t]{4}{*}{10} & $\mathrm{~B}(\mathrm{~PB})$ & 16.16 & 0.74 & 0.38 & 131.76 & 10.50 \\
\hline & $\mathrm{D}(\mathrm{PB})$ & 10.86 & 0.65 & 0.27 & 121.92 & 10.10 \\
\hline & $\mathrm{G}(\mathrm{PB})$ & 6.28 & 0.45 & 0.00 & 86.73 & 10.31 \\
\hline & I (PB) & 9.62 & 0.88 & 0.36 & 44.36 & 8.17 \\
\hline 11 & $\mathrm{~A}(\mathrm{~PB})$ & 3.91 & 0.63 & 0.12 & 54.54 & 6.36 \\
\hline \multirow[t]{2}{*}{12} & $\mathrm{Dx}(\mathrm{BM})$ & 0.00 & 0.21 & 0.00 & 15.65 & 2.56 \\
\hline & $\mathrm{B}(\mathrm{PB})$ & 2.68 & 0.56 & 0.06 & 59.99 & 11.98 \\
\hline \multirow[t]{2}{*}{13} & $\mathrm{G}(\mathrm{PB})$ & 9.06 & 0.70 & 0.39 & 37.19 & 0.82 \\
\hline & $\mathrm{K}(\mathrm{PB})$ & 5.82 & 1.01 & 0.56 & 35.44 & 11.25 \\
\hline \multirow[t]{3}{*}{14} & $\mathrm{Dx}(\mathrm{BM})$ & 0.21 & 0.37 & 0.04 & 11.09 & 1.52 \\
\hline & $\mathrm{C}(\mathrm{PB})$ & 9.65 & 1.11 & 0.41 & 41.99 & 9.76 \\
\hline & $\mathrm{E}(\mathrm{PB})$ & 9.26 & 0.94 & 0.26 & 65.22 & 9.82 \\
\hline \multicolumn{7}{|c|}{ Resistant (R) } \\
\hline 1 & $\mathrm{~A}(\mathrm{~PB})$ & 10.44 & 0.89 & 1.82 & 62.74 & 4.81 \\
\hline 2 & Dx (BM) & 0.27 & 0.20 & 0.00 & 9.93 & 0.46 \\
\hline & $\mathrm{A}(\mathrm{PB})$ & 18.95 & 1.02 & 0.00 & 142.96 & 13.37 \\
\hline & $\mathrm{C}(\mathrm{BM})$ & 1.18 & 0.18 & 0.00 & 14.14 & 0.00 \\
\hline & $\mathrm{F}(\mathrm{PB})$ & 5.23 & 0.40 & 0.00 & 77.62 & 7.25 \\
\hline & $\mathrm{H}(\mathrm{BM})$ & 0.19 & 0.25 & 0.04 & 17.02 & 0.58 \\
\hline 3 & Dx (BM) & 0.00 & 0.39 & 0.15 & 28.20 & 0.00 \\
\hline & $\mathrm{A}(\mathrm{BM})$ & 1.13 & 0.51 & 0.11 & 20.87 & 1.59 \\
\hline 4 & $\mathrm{C}(\mathrm{PB})$ & 3.16 & 0.46 & 0.15 & 67.02 & 5.18 \\
\hline & $\mathrm{G}(\mathrm{PB})$ & 2.50 & 0.79 & 0.51 & 79.61 & 4.59 \\
\hline 5 & $\mathrm{D}(\mathrm{PB})$ & 6.24 & 0.54 & 0.00 & 55.18 & 3.28 \\
\hline & $\mathrm{F}(\mathrm{PB})$ & 27.58 & 0.77 & 1.63 & 123.06 & 0.00 \\
\hline & $\mathrm{H}(\mathrm{PB})$ & 10.54 & 0.44 & 0.00 & 79.79 & 0.00 \\
\hline & $\mathrm{K}(\mathrm{PB})$ & 3.62 & 0.52 & 0.42 & 28.62 & 3.11 \\
\hline & $\mathrm{N}(\mathrm{PB})$ & 4.51 & 0.70 & 0.19 & 113.20 & 18.98 \\
\hline
\end{tabular}

Table I. Continued.

\begin{tabular}{|c|c|c|c|c|c|c|}
\hline \multirow{2}{*}{\multicolumn{2}{|c|}{ Sample }} & \multicolumn{5}{|c|}{ Gene } \\
\hline & & $A B C B 1$ & $A B C C 1$ & \multirow{2}{*}{$\begin{array}{r}A B C G 2 \\
0.38\end{array}$} & \multirow{2}{*}{\begin{tabular}{r}
\multicolumn{1}{l}{ MVP } \\
14.53
\end{tabular}} & \multirow{2}{*}{$\begin{array}{c}S L C 22 A 1 \\
1.22\end{array}$} \\
\hline 6 & $\mathrm{~A}(\mathrm{BM})$ & 1.93 & 0.29 & & & \\
\hline & $\mathrm{C}(\mathrm{BM})$ & 0.33 & 0.27 & 0.07 & 22.23 & 1.38 \\
\hline & $\mathrm{F}(\mathrm{BM})$ & 0.66 & 0.14 & 0.06 & 6.08 & 1.03 \\
\hline \multirow[t]{3}{*}{7} & $\mathrm{~A}(\mathrm{~PB})$ & 50.15 & 1.36 & 4.60 & 200.41 & 0.00 \\
\hline & $\mathrm{D}(\mathrm{PB})$ & 8.42 & 0.70 & 0.27 & 96.24 & 8.89 \\
\hline & $\mathrm{G}(\mathrm{PB})$ & 15.66 & 1.59 & 0.17 & 61.55 & 6.95 \\
\hline \multicolumn{7}{|c|}{ Resistant with BCR-ABL mutations (RM) } \\
\hline \multirow[t]{5}{*}{1} & $\mathrm{C}(\mathrm{PB})$ & 1.09 & 0.33 & 1.13 & 25.62 & 1.36 \\
\hline & $\mathrm{D}(\mathrm{PB})$ & 7.28 & 0.59 & 0.49 & 60.13 & 20.92 \\
\hline & $\mathrm{E}(\mathrm{BM})$ & 0.00 & 0.12 & 0.00 & 18.01 & 0.00 \\
\hline & $\mathrm{G}(\mathrm{PB})$ & 0.00 & 0.44 & 0.00 & 183.88 & 74.56 \\
\hline & I (BM) & 1.22 & 0.56 & 0.17 & 41.44 & 2.15 \\
\hline \multirow[t]{4}{*}{2} & $\mathrm{~A}(\mathrm{~PB})$ & 125.69 & 9.79 & 0.00 & 978.12 & 0.00 \\
\hline & $\mathrm{C}(\mathrm{PB})$ & 59.26 & 1.56 & 1.39 & 77.57 & 6.74 \\
\hline & $\mathrm{F}(\mathrm{PB})$ & 1.60 & 0.16 & 0.09 & 33.47 & 1.90 \\
\hline & $\mathrm{H}(\mathrm{PB})$ & 30.26 & 0.93 & 0.22 & 51.44 & 2.26 \\
\hline \multirow[t]{2}{*}{3} & $\mathrm{H}(\mathrm{PB})$ & 2.20 & 0.36 & 0.30 & 17.69 & 1.12 \\
\hline & $\mathrm{L}(\mathrm{PB})$ & 14.54 & 0.90 & 0.61 & 87.48 & 9.42 \\
\hline \multirow[t]{3}{*}{4} & $\mathrm{Dx}(\mathrm{PB})$ & 0.46 & 0.12 & 0.00 & 7.34 & 0.00 \\
\hline & $\mathrm{C}(\mathrm{BM})$ & 4.70 & 0.14 & 0.00 & 24.74 & 0.00 \\
\hline & $\mathrm{J}(\mathrm{BM})$ & 0.82 & 0.25 & 0.14 & 15.76 & 0.75 \\
\hline \multirow[t]{2}{*}{5} & $\mathrm{Dx}(\mathrm{BM})$ & 0.00 & 0.18 & 0.06 & 9.34 & 1.41 \\
\hline & $\mathrm{A}(\mathrm{BM})$ & 0.86 & 0.68 & 0.25 & 7.79 & 0.16 \\
\hline \multirow[t]{3}{*}{7} & $\mathrm{~A}(\mathrm{~PB})$ & 4.88 & 0.45 & 0.20 & 49.00 & 8.48 \\
\hline & $\mathrm{F}(\mathrm{PB})$ & 10.58 & 0.81 & 0.48 & 37.15 & 9.80 \\
\hline & $\mathrm{H}(\mathrm{PB})$ & 15.82 & 1.27 & 0.28 & 30.51 & 7.05 \\
\hline \multirow[t]{3}{*}{8} & $\mathrm{~B}(\mathrm{~PB})$ & 13.83 & 1.35 & 0.00 & 154.97 & 13.10 \\
\hline & $\mathrm{D}(\mathrm{PB})$ & 13.89 & 0.52 & 0.00 & 24.21 & 2.68 \\
\hline & $\mathrm{G}(\mathrm{PB})$ & 15.66 & 1.04 & 0.15 & 33.17 & 4.65 \\
\hline
\end{tabular}

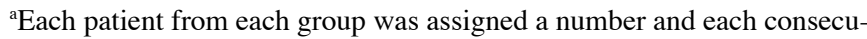
tive sample had a letter in alphabetical order, except for the diagnostic sample (Dx). BM, bone marrow; PB, peripheral blood.

$(\mathrm{p}=0.002), A B C G 2(\mathrm{p}=0.001), M V P(\mathrm{p}=0.006)$ and $S L C 22 A 1$ $(\mathrm{p}=0.005)$; in samples from resistant patients for the $A B C B 1$ gene $(\mathrm{p}=0.031)$ and in samples from patients with mutations for the $M V P$ gene $(\mathrm{p}=0.03)$. For the majority of patients, the gene with the highest expression level was $M V P$ followed by $A B C B 1$ and $S L C 22 A 1$. In contrast, $A B C C 1$ and $A B C G 2$ genes presented with the lowest expression values with low variations throughout the time period (Table I). Concerning only diagnostic samples, we did not observe any significant difference in the expression values of the studied genes between the susceptible and resistant patients.

Transcript expression of $A B C B 1, A B C C 1, A B C G 2, S L C 22 A 1$ and $M V P$ and response to treatment. In the IM-sensitive patient group, the expression levels of the studied genes were more stable compared to both groups of IM-resistant patients whose samples revealed high variations in the levels of expression. Moreover, while in sensitive patients the expression level of $S L C 22 A 1$ was mostly higher compared to $A B C B 1$; in both groups of resistant patients that relationship was reversed (Figs. 2-4). 
A

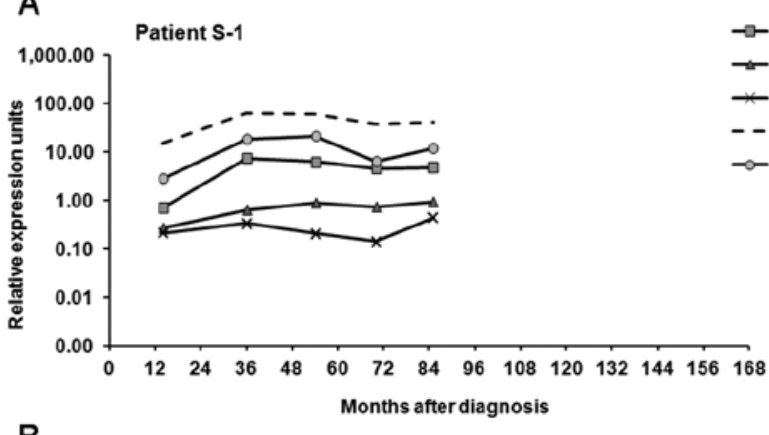

B

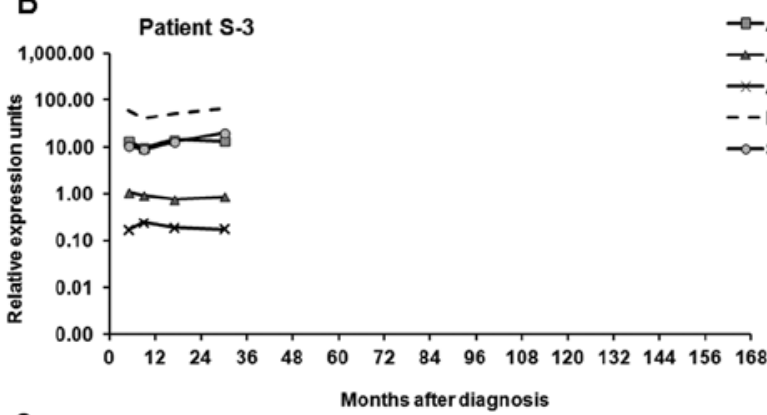

C Patient S-5

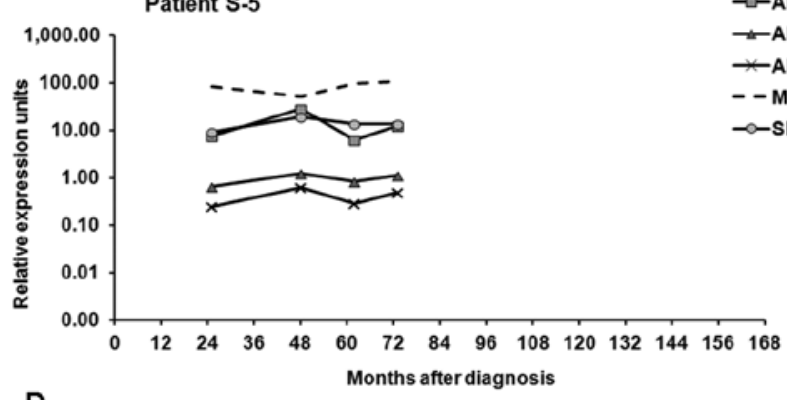

D

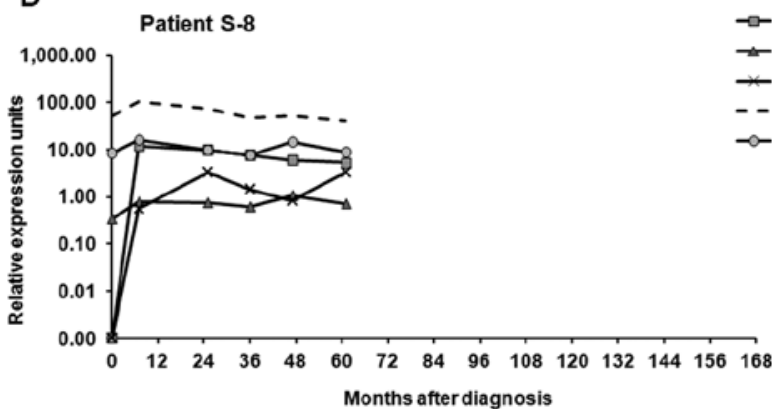

Figure 2. mRNA relative expression values of the studied genes $(A B C B 1$, $A B C C 1, A B C G 2, M V P$ and $S L C 22 A 1)$ over time in illustrative patients sensitive to IM (S): (A) patient S-1, (B) patient S-3, (C) patient S-5, (D) patient S-8.

Considering only the follow-up samples, we discovered that patients susceptible to therapy presented significantly higher values of $S L C 22 A l$ expression ( $\mathrm{S}$ vs. $\mathrm{R}, \mathrm{p}<0.001$; $\mathrm{S}$ vs. $\mathrm{RM}, \mathrm{p}<0.005)$, compared to both groups of patients resistant to IM (Fig. 5). However, for the $A B C B 1, A B C G 2$ and $M V P$ genes there were no significant differences in expression levels between the groups of patients. The $A B C C 1$ gene also presented differences in expression, with higher expression in sensitive patients than in resistant patients $(\mathrm{p}=0.045)$ (Fig. 5).

Correlation of gene expression patterns. Irrespectively of the response to treatment, we observed that a correlation exists

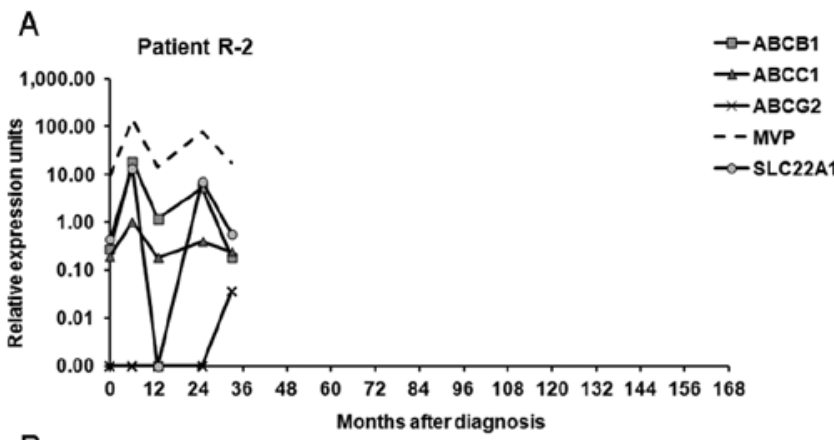

B

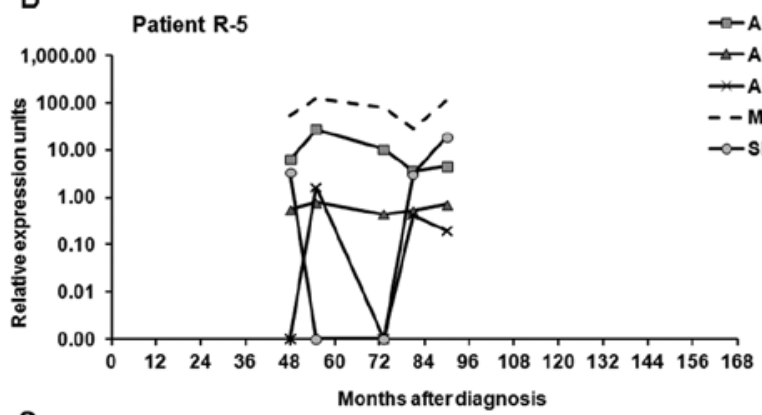

C
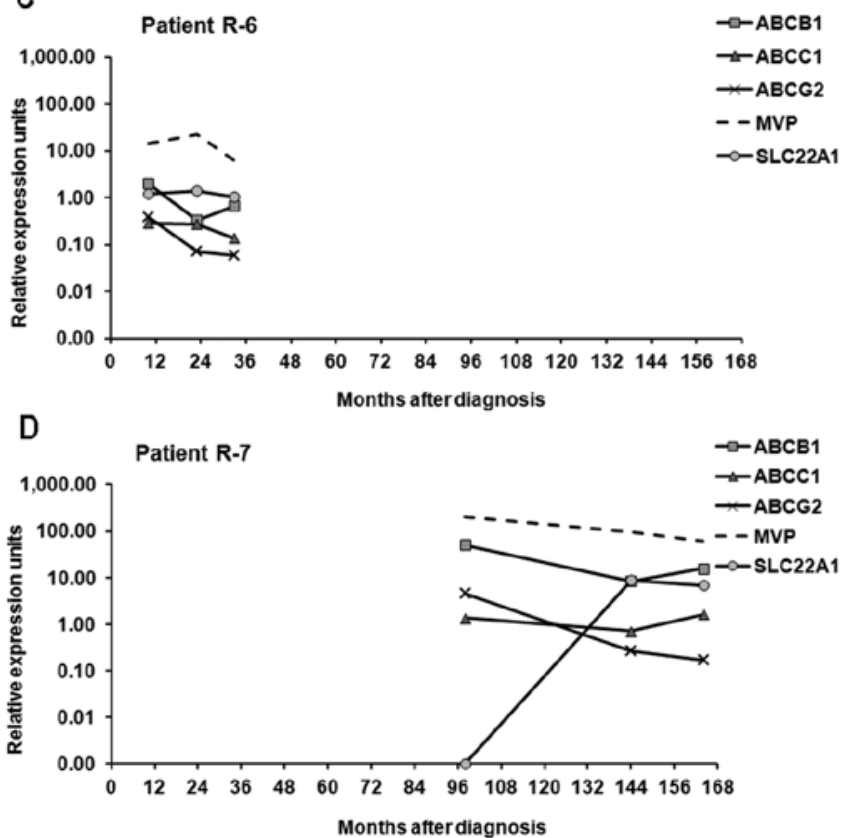

Figure 3. mRNA relative expression values of the studied genes ( $A B C B 1$ $A B C C 1, A B C G 2, M V P$ and $S L C 22 A 1)$ over time in illustrative patients resistant to IM (R): (A) patient R-2, (B) patient R-5, (C) patient R-6, (D) patient R-7.

between the patterns of gene expression over a period of time for the genes included in this study. When the expression of one gene increased, the expression of the other increased concurrently and vice versa (Figs. 2-4). This observation was confirmed using the Spearman's rank correlation coefficient that revealed a significant correlation between the expression of all of the genes, apart from $A B C G 2$ with the genes MVP and SLC22A1. Most correlations were moderate $(0.245-0.520)$ while the correlation between $A B C B 1$ and $A B C C 1$ was strong (0.771).

Mutations. Seven different $B C R-A B L 1$ kinase domain point mutations were identified in the samples from seven resistant 

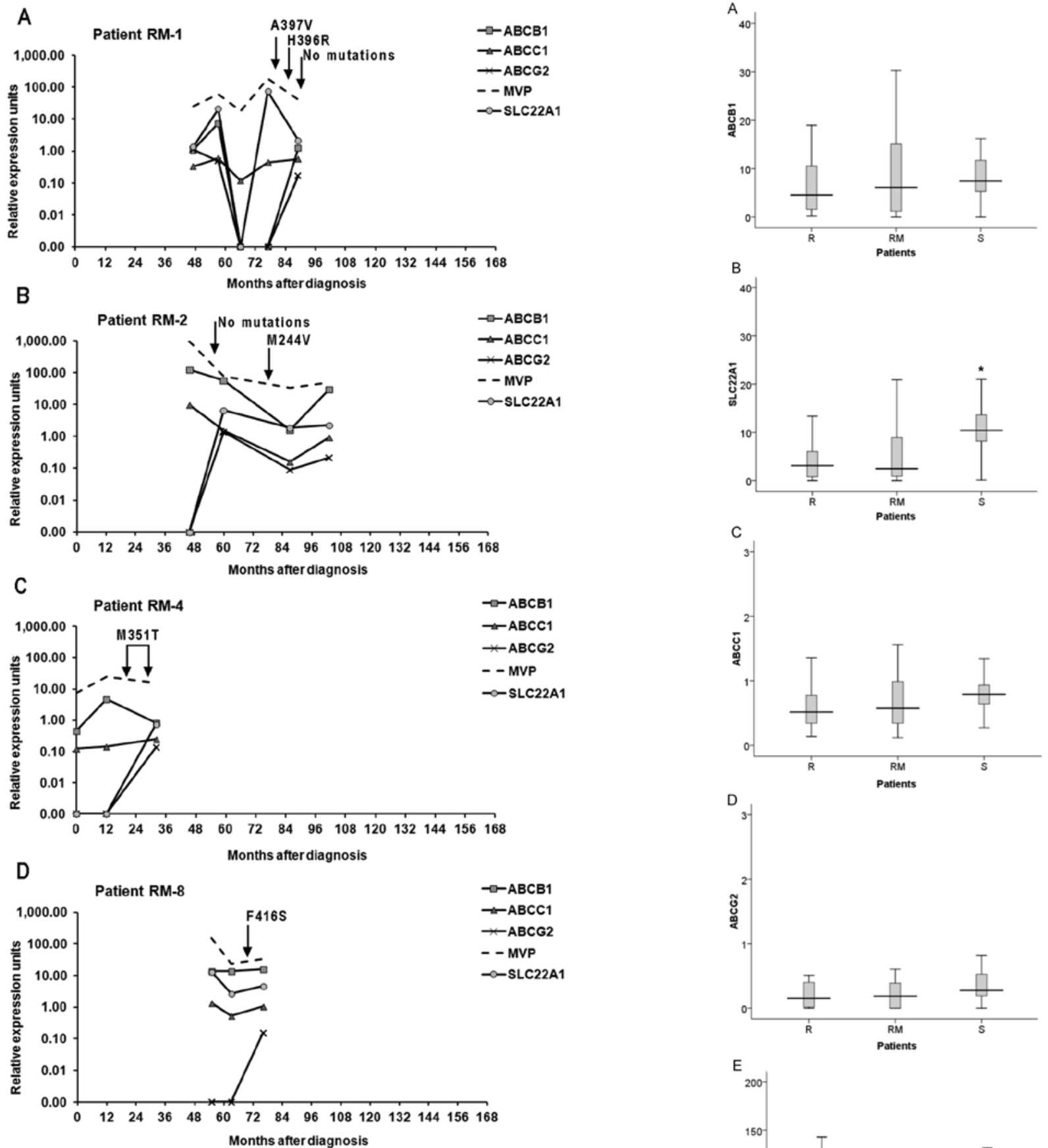

Figure 4. mRNA relative expression values of the studied genes $(A B C B 1$, $A B C C 1, A B C G 2 M V P$ and $S L C 22 A 1)$ over time in illustrative patients resistant to IM with $B C R-A B L$ mutations (RM): (A) patient RM-1, (B) patient RM-2, (C) patient RM-4, (D) patient RM-8. Mutations are indicated by an arrow at the time they were detected.

patients: A397V (RM-1), H396R (RM-1), M244V (RM-2), F317L (RM-3), M351T (RM-4, RM-7), D276G (RM-5) and F416S (RM-8) (data not shown). The mutation M351T was the most frequent; found in two patients. In patient RM-1, two different point mutations were found at different times during disease evolution (Fig. 4A).

Polymorphisms. We also investigated the role of c.1236T $>\mathrm{C}$ ABCB1 and c.480G $>$ C SLC22A1 polymorphisms, both previously described as influencing the treatment outcome and displaying high frequency in the population. However, no

relationship between the genotype and response to treatment was detected (Fisher's test, $\mathrm{p}>0.05$ ) (Fig. 6). 

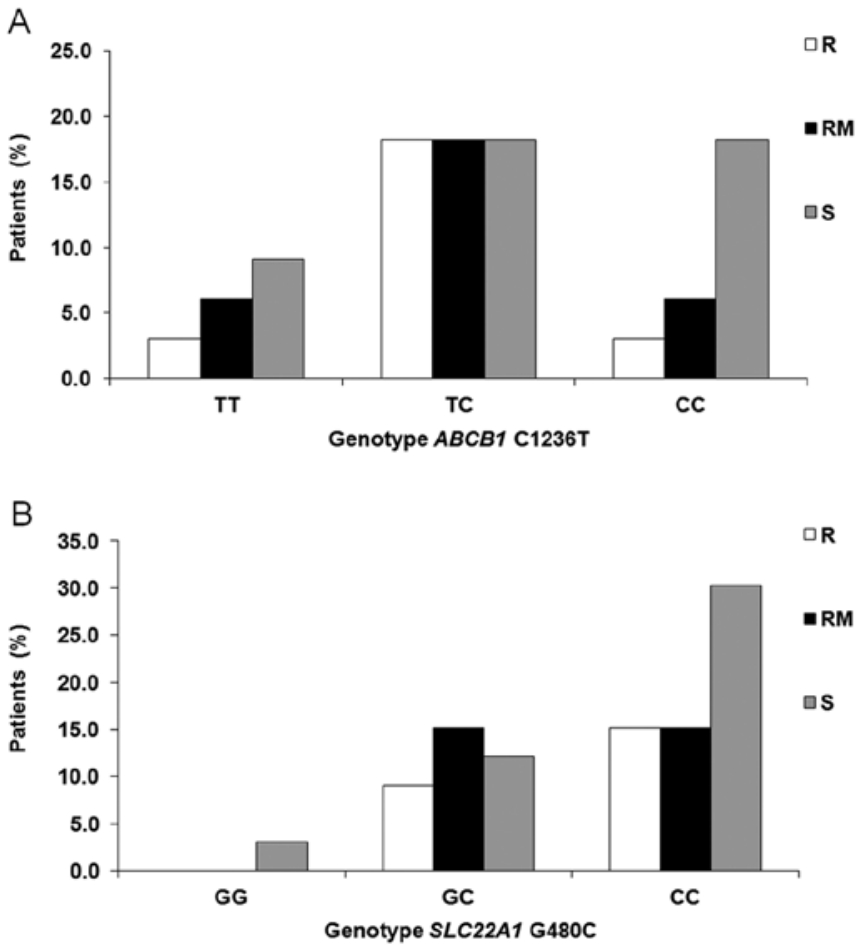

Figure 6. Percentage of patients with the different genotypes stratified by group of patients: sensitive to IM ( $\mathrm{S} ; \mathrm{n}=15)$, resistant to IM $(\mathrm{R} ; \mathrm{n}=8)$ and resistant to IM with identified mutations in the kinase domain of $B C R-A B L 1$ (RM $\mathrm{n}=10$ ). (A) $A B C B 11236 \mathrm{C}>\mathrm{T}$, (B) $S L C 22 A 1480 \mathrm{G}>\mathrm{C}$. No relation between both genotype and response to treatment was found (Fisher's test, $\mathrm{p}>0.05$ ).

\section{Discussion}

The determinants that affect response to imatinib mesylate (IM) remain unknown. However, chronic administration of IM to patients may alter the expression of drug transporters, thus affecting therapeutic success, since IM plasma levels are a critical factor in treatment outcome (9).

During the development of resistance in K562 CML cells, we found that the expression pattern of transporters varied during chronic drug exposure (20). To evaluate whether similar variations occur in vivo in patients treated with IM, we assessed the expression levels of $S L C 22 A 1, A B C B 1, A B C C 1$, $A B C G 2$ and $M V P$ at different time points during treatment in CML patients, who were either responsive or resistant to IM therapy. We observed a significant increase in drug transporters in follow-up samples compared to diagnostic samples, particularly in IM-responsive patients, which is in agreement with the hypothesis that chronic use of IM may induce their expression. In CML patients resistant to IM, bone marrow mononuclear cells demonstrated at least a doubling of $A B C G 2$ and $A B C B 1$ expression after IM exposure (25). In another study, peripheral blood cells from all patients undergoing IM therapy for more than six months expressed several transporters of the ABC family (ABCB1, $A B C C 1, A B C G 2)$ and $L R P$ protein (19). Prolonged in vitro treatment of intestinal epithelial Caco 2 cells with IM specifically upregulated the expression of $A B C G 2$ and $A B C B 1$ and this drug-induced overexpression was a steady phenomenon and stable over time (26). Furthermore, some data do not support the possibility that the upregulation of $\mathrm{ABC}$ transporters contributes to the development of resistance to IM (27) and even demonstrate that IM acts as an environmental factor reducing activity of mechanisms involved in its own clearance (28). Notwithstanding, some observations suggest that several drug transporters may cooperate in the extrusion of IM and may be coordinately regulated (26), providing an important means to protect the body from xenobiotic insults (29).

Analysis of the correlation of expression levels among the genes under study by the Spearman's correlation coefficient demonstrated a significant positive correlation among $A B C B 1$, $A B C C 1, M V P$ and $S L C 22 A 1$. ABCG2 gene expression levels also correlated with $A B C B 1$ and $A B C C 1$, but not with $M V P$ or $S L C 22 A 1$. The observations that these genes are co-expressed may suggest their joint activation as already reported for childhood acute lymphoid leukemia (15). In addition, the co-expression of P-gp and MRP1 was documented in CML patients (18). Our results also revealed a correlation between efflux genes and the influx SLC22Al which supports the hypothesis that absolute bioavailability may also be influenced by the balance between efflux and influx transport (30) and that inter-patient variation in sensitivity is mainly mediated by the balance between the uptake and retention of IM (31).

While responding patients revealed a stable expression in consecutive samples, there was considerable variation in gene expression in resistant patients (Figs. 2-4). The cyclic nature of the variations we observed may reflect the heterogeneity of differentiation in the population of leukemic cells throughout the disease, and the repopulating effect of stem cell division. Division of leukemic stem cells or early precursors is critical for CML progression and such cells are known to express several transporters belonging to the ABC family (32). In a study with acute lymphoid leukemia patients (15) the expression of the MDR genes investigated was also found to be highly variable. Those variations may be due to specific factors and conditions that stimulate their expression, such as cytotoxic agents, thermal shock, genotoxic stress, inflammatory mediators, cytokines and growth factors.

The significant variation we observed in IM-resistant patients requires caution in attempting to associate expression signatures with clinical outcomes or prognostic features. Various studies have been performed to evaluate gene expression signatures with resistance $(33,34)$, but most studies have compared IM responsive cohorts with non-responsive cohorts using single point samples, and there is considerable heterogeneity in the different studies. As our results indicate, expression signatures of IM-resistant patients were not stable and may induce error.

In the current study, $A B C C 1$ expression was low in the patient samples. A low value of ABCC1 expression in $\mathrm{K} 562$ CML cells was also observed (20). ABCC1/MRPl levels did not correlate with treatment outcome in patients with CML (13). However, high levels of $A B C C 1$ mRNA were deemed to predict resistance to IM in myeloid blast crisis (35). Although it was shown in vitro that IM is a substrate for MRP1 (36), the clinical role of $A B C C 1$ overexpression in CML cells remains to be elucidated. Similarly, the relative mRNA expression of $A B C G 2$ was maintained low through time. There has been significant controversy regarding the potential of ABCG2 transporter to confer resistance to IM since it may be either its substrate or 
inhibitor (37). Our results indicate that $A B C G 2$ may indeed have a limited role in IM resistance in clinical samples.

In the studied patient samples, MVP was the gene that attained the highest expression values. This protein was first associated to MDR by Scheffer et al (38) who detected its overexpression in a multidrug-resistant but P-gp-negative lung cancer cell line. Afterwards, MVP overexpression was found in several human cancer cell lines (17). Despite its high expression levels, we did not note any differences between IM-sensitive and IM-resistant patients, making the role of $M V P$ overexpression in drug resistance unclear.

The $A B C B 1$ and SLC22A1 genes also demonstrated high relative expression values, particularly after treatment and the greatest variations throughout time in IM-resistant patients, and the mRNA expression of the SLC22Al gene was significantly higher in sensitive than in resistant patients. Moreover, while in sensitive patients the expression level of $S L C 22 A 1$ was generally higher than $A B C B 1$, in both groups of IM-resistant patients that relation was reversed. The expression values of $S L C 22 A 1$ in diagnostic samples from patients susceptible to therapy tended to be higher than in samples from IM-resistant patients (data not shown). Collectively, these data suggest an important role for $S L C 22 \mathrm{Al}$ expression values in response to treatment. Several studies have suggested that early achievement of high IM intracellular concentrations may be a crucial determinant of cytogenetic response. A significant difference was observed between pre-treatment SLC22A1 mRNA levels in cytogenetic responders (CyR) and non-responders (CyNR) (25). Another study also confirmed that the expression of SLC22A1 is important in determining the clinical response to IM since patients with high pre-treatment expression had superior CCyR rates, progression-free and overall survival (13). A correlation between the level of SLC22Al mRNA and hOCT-1 activity was previously demonstrated $(11,39)$ and it was also shown that only the activity of hOCT1 in mature CML blasts is associated with a therapeutic outcome and not the hOCT1 activity in immature CD $34^{+}$cells (40). Hu et al (41) showed that $S L C 22 A 1$ is significantly interrelated with $A B C B 1, A B C G 2$ and SLCO1A2 and suggested that SLC22A1 gene expression may alternatively be a composite surrogate for the expression of various transporters relevant to the intracellular uptake and retention of IM rather than be the determinant factor.

Several $B C R-A B L 1$ gene mutations detected in our patient samples were already described as conferring partial resistance to IM $(42,43)$ such as H396R located in the activation (A) loop of the protein, M244V in the ATP binding site, F317L in the IM binding site and D276G in the C-helix domain. The M351T mutation located in the catalytic domain presented in patient DRM-4 was previously described as not conferring resistance to IM (43). The mutation F416S, to the best of our knowledge, has not been previously reported and therefore its influence in treatment outcome remains unknown. Although the A-loop mutation $\mathrm{A} 397 \mathrm{~V}$ has also not been previously reported, the substitution of alanine by proline (A397P) in the same codon has been noted several times (44-47). Nevertheless, the insensitivity to IM for both mutations, in the absence of further data, is at present rather a conjecture. None of the mutations found appeared to affect the expression of the genes under study (Fig. 4). Sporadic clones bearing kinase domain mutations do not invariably lead to relapse, and additional factors are required to induce a fully drug-resistant phenotype (48) since they demonstrate high rates of regression, suggesting weak selective effects (49). Other mechanisms must be responsible for resistance and are not necessarily mutually exclusive with mutations. Notwithstanding, in a number of patients, mutations may be simple 'bystanders'. Exceptions are those mutations highly insensitive to IM and those which a molecular mechanism of resistance has been posited e.g., T315I, P-loop mutations and F359V (50), neither of which were found in the patients under study.

The frequency of $B C R-A B L 1$ transcripts $(33.3 \%$ e13a 2 and $48.5 \%$ e14a2) found in the present study is similar to earlier observations $(51,52)$. Although several authors revealed a relation between type of transcript and response to treatment, the results are contradictory $(51,53)$ and others did not note any relation (52). Therefore, it remains controversial, and the clinical significance of the specific $B C R-A B L 1$ transcript among CML patients has not been clearly established. In five of our patients we found the ela 2 transcript, which encodes a 190-kDa protein, in coexistence with e13a2/e14a2. Its expression as the only transcript is rare in CML and is associated with an inferior outcome to therapy with TKIs (54). We were able to reveal it in association with the most prevalent transcripts and in this regard, response to therapy was not worse.

We did not note any significant differences between the three groups of patients, in the frequencies of the analyzed SNPs. Nevertheless, for $A B C B 1$, we found a tendency for a higher incidence of the $1236 \mathrm{CC}$ genotype in patients sensitive to IM therapy (Fig. 6A). Some pharmacogenetic association studies assessing clinical efficacy were performed in CML patients receiving IM therapy. However, data involving the role of pharmacogenetics in response and survival in $\mathrm{CML}$ patients are scarce and scantily reproduced. In a previous study, patients with a 1236TT genotype had higher IM concentrations and a better response (55) and another group observed more resistance in CML patients homozygous for the $1236 \mathrm{~T}$ allele (56). Also steady-state IM clearance was associated with genotype, being higher in 1236TT individuals (28). Regarding the gene $S L C 22 A 1$, we were not able to confirm or reject that CML patients carrying a homozygous GG genotype for the $480 \mathrm{C}>\mathrm{G}$ polymorphism demonstrated a high rate of loss of response or treatment failure to IM therapy (57) (Fig. 6B).

In conclusion, our data indicate that the expression signatures in IM-resistant patients are not stable, but vary significantly over time, advising caution when comparing single point samples from responsive and resistant patients. The data also suggest that the equilibrium between influx and efflux of the cell is important to determine drug response and highlights the possible role of the influx transporter SLC22Al in treatment response, since not only the patients with higher $S L C 22 A 1$ expression attain better treatment outcome but those with lower expression are more likely to develop resistance.

\section{Acknowledgements}

Financial support from Fundação para a Ciência e a Tecnologia (FCT) through the project PTDC/SAU-GMG/71720/2006 and PEst-OE/SAU/UI0009/2011-2012, the grant SFRH/BPD/39046/ 2007 and the Programa Ciência 2008 was provided to M.G. 


\section{References}

1. Druker BJ: Translation of the Philadelphia chromosome into therapy for CML. Blood 112: 4808-4817, 2008.

2. Jabbour E, Cortes J and Kantarjian H: Long-term outcomes in the second-line treatment of chronic myeloid leukemia: a review of tyrosine kinase inhibitors. Cancer 117: 897-906, 2011.

3. Hochhaus A, O'Brien SG, Guilhot F, et al: Six-year follow-up of patients receiving imatinib for the first-line treatment of chronic myeloid leukemia. Leukemia 23: 1054-1061, 2009.

4. de Lavallade H, Apperley JF, Khorashad JS, et al: Imatinib for newly diagnosed patients with chronic myeloid leukemia: incidence of sustained responses in an intention-to-treat analysis. J Clin Oncol 26: 3358-3363, 2008.

5. Marin D, Milojkovic D, Olavarria E, et al: European LeukemiaNet criteria for failure or suboptimal response reliably identify patients with CML in early chronic phase treated with imatinib whose eventual outcome is poor. Blood 112: 4437-4444, 2008.

6. Baccarani M, Castagnetti F, Gugliotta G, Palandri F and Soverini S: Response definitions and European Leukemianet Management recommendations. Best Pract Res Clin Haematol 22: 331-341, 2009.

7. Soverini S, Colarossi S, Gnani A, et al: Contribution of ABL kinase domain mutations to imatinib resistance in different subsets of Philadelphia-positive patients: by the GIMEMA Working Party on Chronic Myeloid Leukemia. Clin Cancer Res 12: 7374-7379, 2006.

8. Larson RA, Druker BJ, Guilhot F, et al: Imatinib pharmacokinetics and its correlation with response and safety in chronic-phase chronic myeloid leukemia: a subanalysis of the IRIS study. Blood 111: 4022-4028, 2008.

9. Picard S, Titier K, Etienne G, et al: Trough imatinib plasma levels are associated with both cytogenetic and molecular responses to standard-dose imatinib in chronic myeloid leukemia. Blood 109: 3496-3499, 2007.

10. Thomas J, Wang L, Clark RE and Pirmohamed M: Active transport of imatinib into and out of cells: implications for drug resistance. Blood 104: 3739-3745, 2004.

11. White DL, Saunders VA, Dang P, et al: Most CML patients who have a suboptimal response to imatinib have low OCT-1 activity: higher doses of imatinib may overcome the negative impact of low OCT-1 activity. Blood 110: 4064-4072, 2007.

12. Clark RE, Davies A, Pirmohamed M and Giannoudis A: Pharmacologic markers and predictors of responses to imatinib therapy in patients with chronic myeloid leukemia. Leuk Lymphoma 49: 639-642, 2008.

13. Wang L, Giannoudis A, Lane S, Williamson P, Pirmohamed M and Clark R: Expression of the uptake drug transporter hOCT1 is an important clinical determinant of the response to imatinib in chronic myeloid leukemia. Clin Pharmacol Ther 83: 258-264, 2008.

14. Rodrigues AS, Dinis J, Gromicho M, Martins C, Laires A and Rueff J: Genomics and cancer drug resistance. Curr Pharm Biotechnol 13: 651-673, 2012.

15. Cortez MAA, Scrideli CA, Yunes JA, et al: mRNA expression profile of multidrug resistance genes in childhood acute lymphoblastic leukemia. Low expression levels associated with a higher risk of toxic death. Pediatr Blood Cancer 53: 996-1004, 2009.

16. Raaijmakers MHGP: ATP-binding-cassette transporters in hematopoietic stem cells and their utility as therapeutical targets in acute and chronic myeloid leukemia. Leukemia 21: 2094-2102, 2007.

17. Steiner E, Holzmann K, Elbling L, Micksche M and Berger W: Cellular functions of vaults and their involvement in multidrug resistance. Curr Drug Targets 7: 923-934, 2006.

18. Vasconcelos FC, Silva KL, Souza PSD, et al: Variation of MDR protein expression and activity levels according to clinical status and evolution of CML patients. Cytometry B Clin Cytom 80: $158-166,2010$

19. Stromskaya T, Rybalkina EY, Kruglov S, et al: Role of p-glycoprotein in evolution of populations of chronic myeloid leukemia cells treated with imatinib. Biochemistry (Mosc) 73: 29-37, 2008.

20. Gromicho M, Dinis J, Magalhães M, et al: Development of imatinib and dasatinib resistance: dynamics of expression of drug transporters ABCB1, ABCC1, ABCG2, MVP, and SLC22A1. Leuk Lymphoma 52: 1980-1990, 2011.

21. Nijmeijer BA, Szuhai K, Goselink HM, et al: Long-term culture of primary human lymphoblastic leukemia cells in the absence of serum or hematopoietic growth factors. Exp Hematol 37: $376-385,2009$
22. van Dongen JJ, Macintyre EA, Gabert JA, et al: Standardized RT-PCR analysis of fusion gene transcripts from chromosome aberrations in acute leukemia for detection of minimal residual disease. Report of the BIOMED-1 Concerted Action: investigation of minimal residual disease in acute leukemia. Leukemia 13: 1901-1928, 1999.

23. Gabert J, Beillard E, van der Velden VHJ, et al: Standardization and quality control studies of 'real-time' quantitative reverse transcriptase polymerase chain reaction of fusion gene transcripts for residual disease detection in leukemia - a Europe Against Cancer program. Leukemia 17: 2318-2357, 2003.

24. Schefe JH, Lehmann KE, Buschmann IR, Unger T and FunkeKaiser H: Quantitative real-time RT-PCR data analysis: current concepts and the novel 'gene expression's CT difference' formula. J Mol Med 84: 901-910, 2006.

25. Crossman LC, Druker BJ, Deininger MWN, Pirmohamed M, Wang L and Clark RE: hOCT 1 and resistance to imatinib. Blood 106: 1133-1134, 2005.

26. Burger $\mathrm{H}$, van Tol H, Brok M, et al: Chronic imatinib mesylate exposure leads to reduced intracellular drug accumulation by induction of the ABCG2 (BCRP) and ABCB1 (MDR1) drug transport pumps. Cancer Biol Therapy 4: 747-752, 2005.

27. Gardner ER, Sparreboom A, Verweij J and Figg WD: Lack of $\mathrm{ABC}$ transporter autoinduction in mice following long-term exposure to imatinib. Cancer Biol Therapy 7: 412-415, 2008.

28. Gurney H, Wong M, Balleine RL, et al: Imatinib disposition and ABCB1 (MDR1, P-glycoprotein) genotype. Clin Pharm Ther 82: 33-40, 2007.

29. Xu C, Li CY-T and Kong A-NT: Induction of phase I, II and III drug metabolism/transport by xenobiotics. Arch Pharm Res 28: 249-268, 2005

30. Eechoute K, Sparreboom A, Burger H, et al: Drug transporters and imatinib treatment: implications for clinical practice. Clin Cancer Res 17: 406-415, 2011.

31. White DL, Saunders VA, Dang P, et al: OCT-1-mediated influx is a key determinant of the intracellular uptake of imatinib but not nilotinib (AMN107): reduced OCT-1 activity is the cause of low in vitro sensitivity to imatinib. Blood 108: 697-704, 2006.

32. de Grouw EPLM, Raaijmakers MHGP, Boezeman JB, et al: Preferential expression of a high number of ATP binding cassette transporters in both normal and leukemic CD $34^{+} \mathrm{CD} 38^{-}$cells. Leukemia 20: 750-754, 2006.

33. de Lavallade H, Finetti P, Carbuccia N, et al: A gene expression signature of primary resistance to imatinib in chronic myeloid leukemia. Leukemia Res 34: 254-257, 2010.

34. Villuendas R, Steegmann JL, Pollán M, et al: Identification of genes involved in imatinib resistance in CML: a gene-expression profiling approach. Leukemia 20: 1047-1054, 2006.

35. Lange T, Günther C, Köhler T, et al: High levels of BAX, low levels of MRP-1, and high platelets are independent predictors of response to imatinib in myeloid blast crisis of CML. Blood 101: 2152-2155, 2003

36. Czyzewski K and Styczynski J: Imatinib is a substrate for various multidrug resistance proteins. Neoplasma 56: 202-207, 2009.

37. Shukla S, Sauna ZE and Ambudkar SV: Evidence for the interaction of imatinib at the transport-substrate site(s) of the multidrug-resistance-linked $\mathrm{ABC}$ drug transporters $\mathrm{ABCB} 1$ (P-glycoprotein) and ABCG2. Leukemia 22: 445-447, 2008.

38. Scheffer GL, Schroeijers AB, Izquierdo MA, Wiemer EA and Scheper RJ: Lung resistance-related protein/major vault protein and vaults in multidrug-resistant cancer. Curr Opin Oncol 12: 550-556, 2000.

39. White DL, Dang P, Engler J, et al: Functional activity of the OCT-1 protein is predictive of long-term outcome in patients with chronic-phase chronic myeloid leukemia treated with imatinib. J Clin Oncol 28: 2761-2767, 2010.

40. Engler JR, Frede A, Saunders VA, Zannettino ACW, Hughes TP and White DL: Chronic myeloid leukemia CD34 ${ }^{+}$cells have reduced uptake of imatinib due to low OCT-1 activity. Leukemia 24: 765-770, 2010.

41. Hu S, Franke RM, Filipski KK, et al: Interaction of imatinib with human organic ion carriers. Clin Cancer Res 14: 3141-3148, 2008.

42. O'Hare T, Eide CA and Deininger MWN: Bcr-Abl kinase domain mutations, drug resistance, and the road to a cure for chronic myeloid leukemia. Blood 110: 2242-2249, 2007.

43. Redaelli S, Piazza R, Rostagno R, et al: Activity of bosutinib, dasatinib, and nilotinib against 18 imatinib-resistant BCR/ABL mutants. J Clin Oncol 27: 469-471, 2008. 
44. Wang L, Knight K, Lucas $\mathrm{C}$ and Clark RE: The role of serial $\mathrm{BCR}-\mathrm{ABL}$ transcript monitoring in predicting the emergence of BCR-ABL kinase mutations in imatinib-treated patients with chronic myeloid leukemia. Haematologica 91: 235-239, 2006.

45. Gruber FX, Lundán T, Goll R, et al: BCR-ABL isoforms associated with intrinsic or acquired resistance to imatinib: more heterogeneous than just ABL kinase domain point mutations? Med Oncol 29: 219-226, 2012.

46. Guilhot F, Apperley J, Kim D-W, et al: Dasatinib induces significant hematologic and cytogenetic responses in patients with imatinib-resistant or -intolerant chronic myeloid leukemia in accelerated phase. Blood 109: 4143-4150, 2007.

47. Ernst T, Hoffmann J, Erben P, et al: ABL single nucleotide polymorphisms may masquerade as BCR-ABL mutations associated with resistance to tyrosine kinase inhibitors in patients with chronic myeloid leukemia. Haematologica 93: 1389-1393, 2008.

48. Willis SG, Lange T, Demehri S, et al: High-sensitivity detection of BCR-ABL kinase domain mutations in imatinib-naive patients: correlation with clonal cytogenetic evolution but not response to therapy. Blood 106: 2128-2137, 2005.

49. Jones D, Chen SS, Jabbour E, Rios MB, Kantarjian H and Cortes J: Uncommon BCR-ABL kinase domain mutations in kinase inhibitor-resistant chronic myelogenous leukemia and $\mathrm{Ph}^{+}$acute lymphoblastic leukemia show high rates of regression, suggesting weak selective effects. Blood 115: 5428-5429, 2010

50. Soverini S, Hochhaus A, Nicolini FE, et al: Bcr-Abl kinase domain mutation analysis in chronic myeloid leukemia patients treated with tyrosine kinase inhibitors: recommendations from an expert panel on behalf of European LeukemiaNet. Blood 118 $1208-1215,2011$
51. Sharma P, Kumar L, Mohanty S and Kochupillai V: Response to imatinib mesylate in chronic myeloid leukemia patients with variant BCR-ABL fusion transcripts. Ann Hematol 89: 241-247, 2010.

52. Polampalli S, Choughule A,Negi N, et al: Analysis and comparison of clinicohematological parameters and molecular and cytogenetic response of two Bcr/Abl fusion transcripts. Genetics Mol Res 7: $1138-1149,2008$

53. Lucas CM, Harris RJ, Giannoudis A, et al: Chronic myeloid leukemia patients with the e13a2 BCR-ABL fusion transcript have inferior responses to imatinib compared to patients with the e14a2 transcript. Haematologica 94: 1362-1367, 2009.

54. Verma D, Kantarjian HM, Jones D, et al: Chronic myeloid leukemia (CML) with P190 BCR-ABL: analysis of characteristics, outcomes, and prognostic significance. Blood 114: 2232-2235, 2009.

55. Dulucq S, Bouchet S, Turcq B, et al: Multidrug resistance gene (MDR1) polymorphisms are associated with major molecular responses to standard-dose imatinib in chronic myeloid leukemia. Blood 112: 2024-2027, 2008.

56. Ni L-N, Li J-Y, Miao K-R, et al: Multidrug resistance gene (MDR1) polymorphisms correlate with imatinib response in chronic myeloid leukemia. Med Oncol 28: 265-269, 2011.

57. Kim DHD, Sriharsha L, Xu W, et al: Clinical relevance of a pharmacogenetic approach using multiple candidate genes to predict response and resistance to imatinib therapy in chronic myeloid leukemia. Clin Cancer Res 15: 4750-4758, 2009. 